\title{
Effect of Metal Film, Thickness and Various Solvents on the SPR Biosensor Sensitivity for illegal Compound Detection
}

\section{SUHERMAN ${ }^{1 *}$, MD. MOTAHAR HOSSAIN ${ }^{2}, \mathrm{KINICHI} \mathrm{MORITA}^{3}$ and TOSHIKAZU KAWAGUCHI ${ }^{4}$}

${ }^{1}$ Department of Chemistry, Faculty of Mathematics and Natural Science, Universitas Gadjah Mada, Sekip Utara Kotak Pos 21 BLS, Yogyakarta 55281, Indonesia.

${ }^{2}$ Department of Chemistry, Faculty of Science, Rajshahi University, Rajshahi-6205, Bangladesh. ${ }^{3}$ New Business Development Office, Corporate R \& D Division, Ushio INC. 1-6-5 Marunouchi, Chiyoda-ku, Tokyo 100-8150, Japan.

${ }^{4}$ Global Station for Food, Land and Water Resources, Global Institution for Collaborative Research and Education, Hokkaido University Kita 9 Nishi 10 Kita-ku, Sapporo, Hokkaido 060-8589, Japan.

*Corresponding author E-mail: suherman.mipa@ugm.ac.id

http://dx.doi.org/10.13005/ojc/340322

(Received: March 21, 2018; Accepted: May 02, 2018)

\begin{abstract}
The development of sensitive surface plasmon resonance (SPR) biosensor for the detection of illegal compound (clenbuterol) was conducted. For the construction of SPR sensor surface, the influence of gold film thickness variations toward resonance angle shift was investigated in order to optimize the metal film effect on the sensitivity of detection. In addition, for the construction of SPR sensor surface, the various solvents used in the dilution of thiol compound, antigen and blocking agent of ethanolamine, were examined. From the gold film thickness variation conducted by sputtering deposition method, it was found that the best thickness for SPR sensor surface was 45-50 nm. This film thickness produce the best SPR angle shift profile with better signal to noise ratio (SNR). Moreover, the best solvents combination for the dilution of dithiobis (succinimidyl propionate) (DSP thiol), clenbuterol antigen and ethanolamine blocking agent were methanol and PBS buffer solution; respectively. By using this combination of solvents, SPR sensor surface could be used for multiple analyses up to 100 times by using $0.2 \mathrm{M} \mathrm{NaOH}$ for surface regeneration process. Clenbuterol was investigated as target compound in this study due to massive used in the livestock (pigs, cows, etc.) by farmers for improving the profit, illegally. The illegal compound was detected by indirect competitive inhibition method. By comparing several thickness of gold film in the construction of the SPR sensor surface, $45-50 \mathrm{~nm}$ metal film thickness shows the better sensitivity by $5 \mathrm{ppt}$.
\end{abstract}

Keywords: Surface plasmon resonance, Metal film thickness, Clenbuterol, Indirect competitive inhibition method.

\section{INTRODUCTION}

Environmental problems are one of major global challenges faced by human beings beside energy shortage ${ }^{1}$. Pollution, climate change, global warming, deforestation, overpopulation, water conservation, and food borne illnesses are common environmental problem ${ }^{2-11}$ in all parts of the world,

This is an 2 Open Access article licensed under a Creative Commons Attribution-Non Commercial-Share Alike 4.0 International License (https://creativecommons.org/licenses/by-nc-sa/4.0/), which permits unrestricted Non Commercial use, distribution and reproduction in any medium, provided the original work is properly cited. 
and the victims in terms of human life and suffering is enormous. The problem in food safety also creates an enormous burden on the economy ${ }^{12}$. An effective environmental policy requires assessment and monitoring of the risks ${ }^{13}$ to consumer health associated with contaminants in raw materials, farming practices, and food processing activities. In this point of view, biosensors development becoming important sector that requires great attention from researchers to ensure the environmental quality such as food safety in all aspects. Biosensors can be considered as a subgroup of chemical sensors in which a biological mechanism is used for analyte detection ${ }^{14-16}$. The International Union of Pure and Applied Chemistry (IUPAC) defines biosensor as a self-contained integrated device that is capable of providing specific quantitative or semi-quantitative analytical information using a biological recognition element (biochemical receptor), which is retained in direct spatial contact with the transduction element ${ }^{17}$.

For environmental applications, the main advantages offered by biosensors upon conventional analytical techniques are the possibility of portability, miniaturization, work on-site, and the ability to measure pollutants in complex matrices with minimal sample preparation ${ }^{18-20}$. Although many of the developed systems cannot compete yet with conventional analytical methods in terms of accuracy and reproducibility, regulatory authorities and industry can use these systems to provide enough information for routine testing and screening of samples considering their biological basis makes them ideal for toxicological measurement ${ }^{21-23}$. For this reason, biosensors are being developed and utilized for monitoring in environmental samples, while conventional techniques can only measure concentrations ${ }^{24}$.

$\beta$-Agonists are usually used as drugs for the treatment of respiratory diseases and asthma ${ }^{25-30}$. However, due to their potential roles in reducing animal fat levels and increasing the amount of muscling in livestock, $\beta$-agonists were used in animals as growth promoters to increase the daily weight gain. $\beta$-agonists compounds can be easily stored in human tissues after meat consumption, and result in many serious health problems with symptoms such as palpitations, tremors and tachipnoea ${ }^{31-33}$.
Recently, more than 150 countries have strictly banned the use of $\beta$-agonists in stockbreeding, including China, Japan and Europe countries, due to their negative side effects in the human body ${ }^{34-36}$. Biosensors are effective technology for detecting chemical and biological analytes since the device incorporating with biologicals or biologically derived sensing element either intimately associated with or integrated within a physicochemical transducer. The usual aim in the biosensors devices is to produce a digital electronic signal, which is proportional to the concentration of a specific analyte or group of analytes.

Surface plasmon resonance (SPR) is mass transducer based on surface plasmon phenomena. SPR can measure the refractive index changes occurring at the interface caused by the binding of target analytes with biointerfacial materials (1 mdeg $\sim 10 \mathrm{RU} \sim 1 \mathrm{ng} / \mathrm{cm}^{2}$ ). In our SPR system, the LOD can be reached up to $30 \mathrm{pg} / \mathrm{cm}^{2}(0.3 \mathrm{RU})$. SPR monitors the binding interactions between a biomolecule (antibody) immobilized on a transducer surface with its biospecific partner (analyte) in solution without the need of labeling biomolecules by exploiting the interfacial refractive index changes associated with any affinity binding interaction. Since its introduction in early $1990 \mathrm{~s}^{37-39}$, SPR plays a central role in the research of biomaterial characterization, kinetics of antibody-analyte interactions in drug discovery and detection of a variety of chemical and biological substances ${ }^{40-47}$.

In principle, SPR measures the change of the dielectric constant occurring at the interface. There are two important components in the SPR system: a) optical setup for the excitation and interrogation of surface plasmons, b) biomolecular recognition elements, which are immobilized on the sensor surface. The resonance of surface plasmons with evanescent field based on total internal reflection occurs when incident beam of p-polarized light strike an electrically conducting metal layer at the interface of a glass chip. Several techniques were commonly used to couple evanescent wave into surface plasmon polariton (SPP), and these techniques known as the configuration of SPR. 


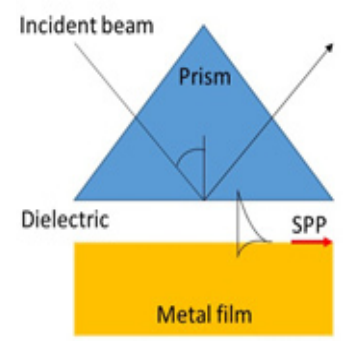

(a)

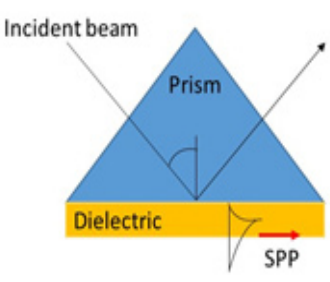

(b)

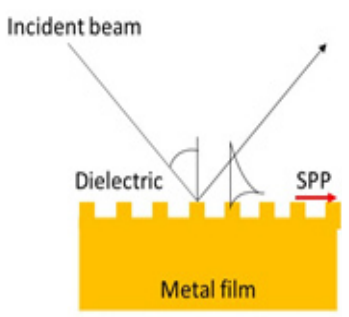

(c)

Fig. 1. Surface plasmon resonance configurations: (a) Otto configuration, (b) Kretschmann configuration, and (c) Diffraction grating

In practical application, Kretschmann configuration was preferable to use because Otto and diffraction grating configurations require high nano-technology to set-up the dielectric medium gap and diffraction grating surface in nano meter order.

If a thin metal film is positioned at the interface between two media, the evanescent wave of incident light is able to interact with free electrons (plasmons) in the metal film at a narrow angle range of incident light (SPR angle). The interaction between an evanescent wave and a surface plasmon in the attenuated total reflection method can be described using the Fresnel theory, represented by Fresnel's equation to estimate the reflection coefficients and transmission coefficients.

$r_{I I}=\frac{\tan \left(\theta_{i}-\theta_{i}\right)}{\tan \left(\theta_{i}+\theta_{i}\right)}$ (Paralle1 reflection)

$r_{1}=-\frac{\sin \left(\theta_{i}-\theta_{i}\right)}{\sin \left(\theta_{i}+\theta_{t}\right)}$ (Perpendicular reflection)

$t_{I I}=\frac{2 \sin \theta_{t} \cos \theta_{i}}{\sin \left(\theta_{i}+\theta_{t}\right) \cos \left(\theta_{i}-\theta_{t}\right)}$ (Paralle1 transmission)

$t_{\perp}=\frac{2 \sin \theta_{t} \cos \theta_{i}}{\sin \left(\theta_{i}+\theta_{t}\right)}$ (Perpendicular transmission)

When this surface plasmon is resonantly excited with the evanescent wave of the incident light, a longitudinal charge density wave propagates along the interface between two different media. In this situation, light energy is lost to the metal film and the intensity of reflected light decreases. From the resonance phenomena, the wave number of evanescent equal to wave number of surface. plasmon.

$$
k_{\text {eva }}=\frac{2 \pi}{\lambda} n_{\text {prism }} \sin \theta
$$

$k_{s p}=\frac{2 \pi}{\lambda} \sqrt{\frac{\varepsilon_{A u} \cdot \varepsilon_{a d-l a y e r}}{\varepsilon_{A u}+\varepsilon_{a d-l a v e r}}}$

$$
k_{e v a}=k_{s p}
$$$$
\frac{2 \pi}{\lambda} n_{\text {prism }} \sin \theta=\frac{2 \pi}{\lambda} \sqrt{\frac{\varepsilon_{t u} \cdot \varepsilon_{\text {ad lager }}}{\varepsilon_{A u}+\varepsilon_{\text {ad-lager }}}}
$$

$$
\theta=\frac{1}{n_{\text {prism }}} \sin ^{-1} \sqrt{\frac{\varepsilon_{A u} \cdot \varepsilon_{\text {ad-layer }}}{\varepsilon_{A u}+\varepsilon_{a d-\text { lajer }}}}
$$

\section{MATERIALS AND METHOD}

\section{Materials}

Potassium hydroxide and ethanol (99.5\%) were obtained from WAKO, Japan, while sodium hydroxide was from Junsei chemical Co., Ltd., Japan. Dithiobis succinimidyl propionate (DSP) and phosphate buffer saline (PBS) were purchased from Sigma Aldrich, USA. Methanol (99\%) was obtained from Dojindo, Japan. Perchloric acid was purchased from Kanto Chemicals, Japan. Clenbuterol hydrochloride and monoclonal mouse $\lg G$ antibody of clenbuterol (Ab) were ordered from LKT laboratories, Inc., USA and Novus Biologicals, USA, respectively. Refractive index matching fluid (refractive index $=1.518$ ) was obtained from Cargille Labs., USA. All chemicals were of reagent or higher grade, and water $(18.2 \mathrm{M} \Omega \mathrm{cm})$ from a Millipore system was used in all experiments.

\section{Method}

In this research, SPR experiments were performed on SPR-670 (Nippon Laser Electronics, 
Japan) equipped with a fully automated flow system consisting of a plunger pump and an injector (Fig. 3). The Au-chip was mounted on the semi-cylindrical prism with a refractive index matching liquid. Red light $(670 \mathrm{~nm})$ emitted from $\mathrm{Ni}-\mathrm{Cd}$ laser was reflected at the Au-coated glass plate at attenuated total reflection angles, and the reflected light intensity was recorded using CCD camera. The reflectance angle, at which the light intensity was minimum (SPR angle), was recorded with time. All the experiments were conducted in an air-conditioned room $\left(25^{\circ} \mathrm{C}\right)$.

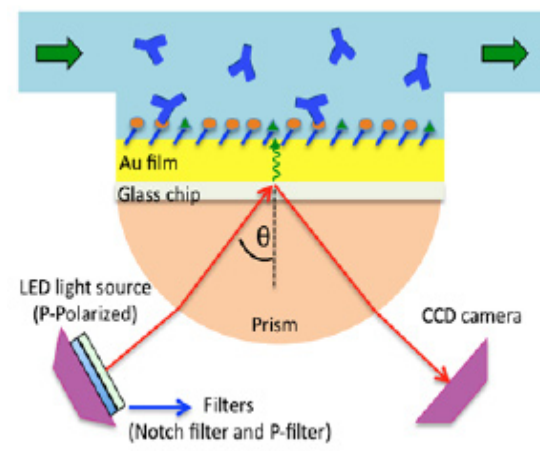

(a)

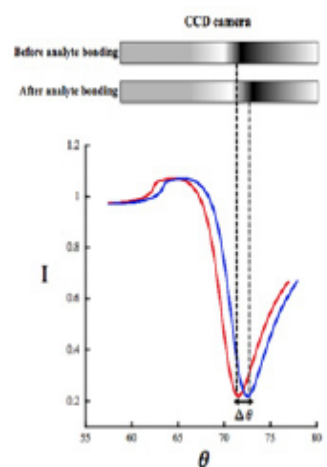

(b)

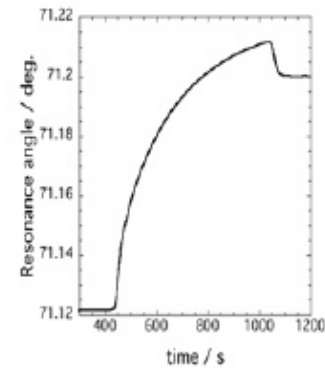

(c)

Fig. 2. Schematic representation of SPR biosensor: (a) optical set-up of SPR sensor combined with flow system, (b) CCD camera before and after the analyte binding, (c) SPR response versus time
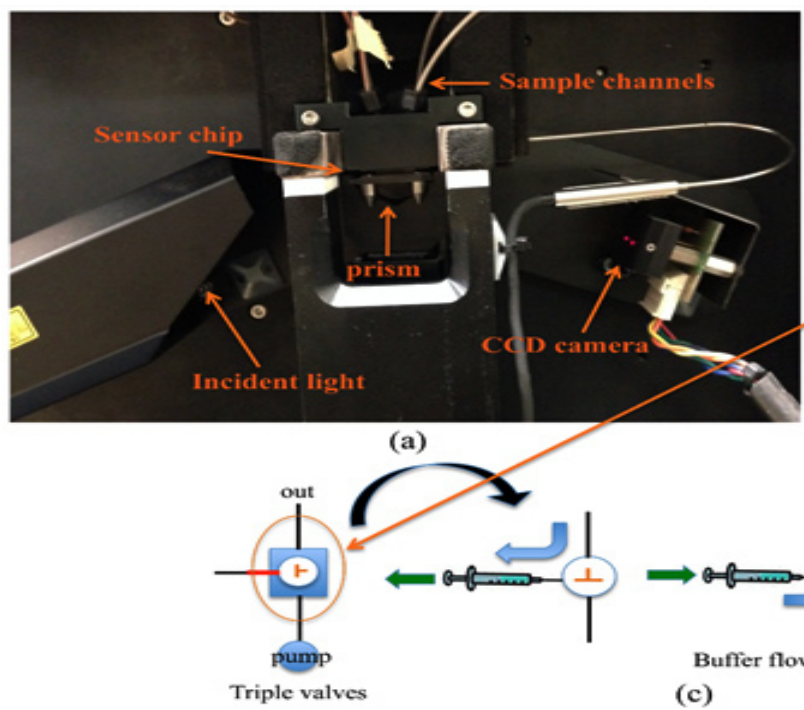

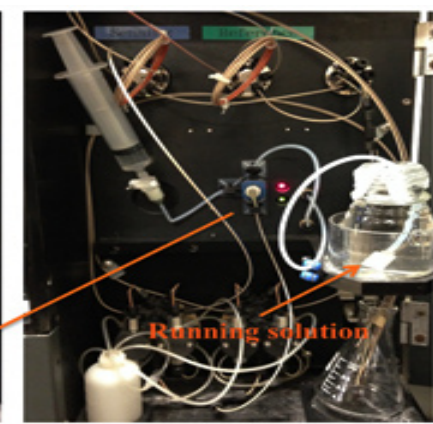

(b)

Fig. 3. SPR 670 from Nippon Laser and Electronics-Japan: (a) sensing part, (b) SPR solution part including plunger and peristaltic pumps, (c) flow system

STM experiments were conducted using a NanoScope STM III (Digital Instruments, USA) operating in the constant current mode (a few hundred picoampere) with the bias voltage typically between 0.5 and $1 \mathrm{~V}$. For imaging, Pt/Ir (80/20) mechanically cut tips (diameter $=0.25 \mathrm{~mm}$, Bruker, USA) were used to scan the surface. 


\section{RESULTS AND DISCUSSION}

Surface plasmon resonance was known as mass transducer which is calculated direct/indirectly the dielectric constant of the interface system. By inserting number of dielectric constants; $\varepsilon A u-50 \mathrm{~nm}$ $=0.04$, emethanol $=32.7$ and refractive index of prism $=1.62$, the following curve (almost linear) was achieved (Figure 4).

Therefore, the dielectric constants change at the interface directly affected on the SPR angle. As the analyte binds to the the sensor surface, the refractive index and SPR angle changes strongly correlated to the increase of the mass

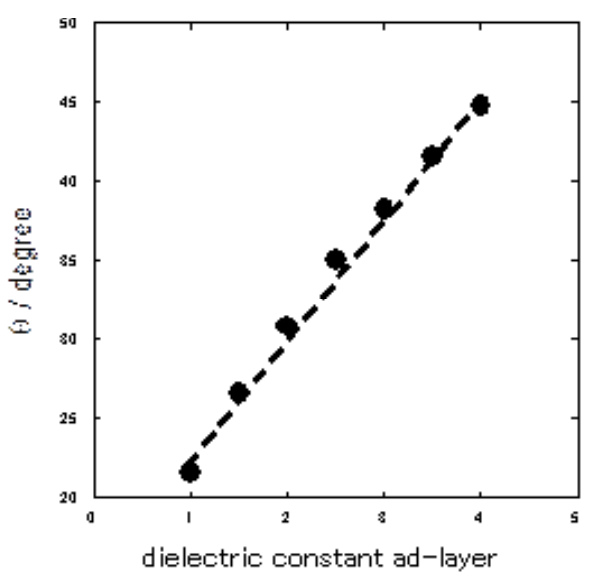

Fig. 4. Dielectric constant change of ad-layer affected on the SPR angle

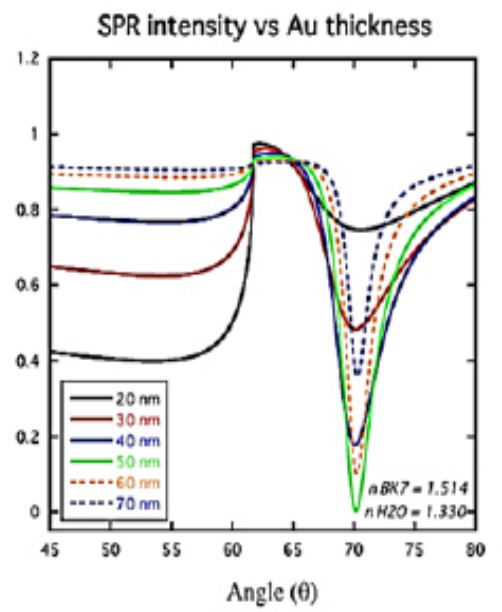

(a) at the sensor surface. A sensorgram can be obtained in real time by plotting the SPR signal against time when the interaction between analyte and the immobilized receptor occurs at the sensor surface ${ }^{48}$.

From the experiment studied the metal thickness effect on the SPR angle shift, the data as mentioned in Fig. 5 below.

The Figure above showed that the metal thickness of 450-500 seconds (about 45-50 nm) produced the best SPR angle shift for the analysis by better sharp angle and higher intensity as predicted by SPR Lab-View simulation program. Fig. 6 showed the solution effect on SPR sensorgram profile as mentioned below.

From the above Figure, it was noticed that the changing of SPR solution type from methanol to the PBS buffer solution affected on the SPR angle $(\Delta \theta)$ for about $900-1000$ mdeg. The experimental result just in accordance with the data taken from SPR Lab-View program (Figure 6).

In the detection process, indirect competitive inhibition method was used to evaluate the metal film thickness effect (Fig. 7). SPR sensor surface constructed from $45-50 \mathrm{~nm}$ gold film thickness produce better sensitivity with 5 ppt than $20 \mathrm{~nm}$ metal film thickness. Furthermore, $45-50 \mathrm{~nm}$ metal film thickness revealed the better SPR angle shift $(\Delta \theta)$ than $20 \mathrm{~nm}$ with the highest SPR angle shift in the detection process at 41.0 mdeg.

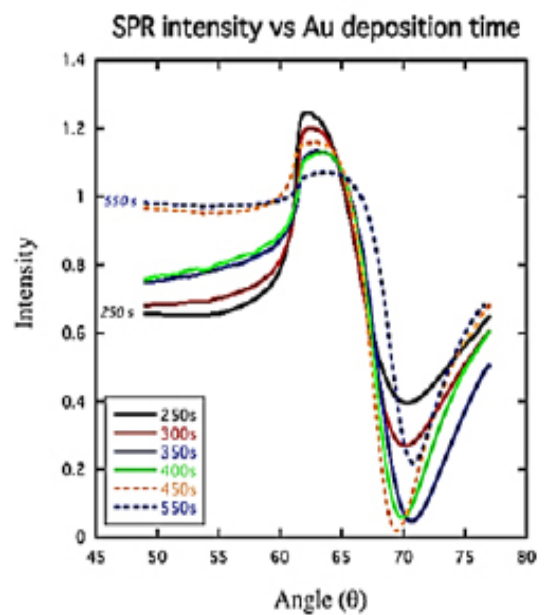

(b)

Fig. 5. SPR intensity affected by Au metal thickness for (a) SPR Lab-View simulation program; and (b) The Au surface prepared by sputtering machine 


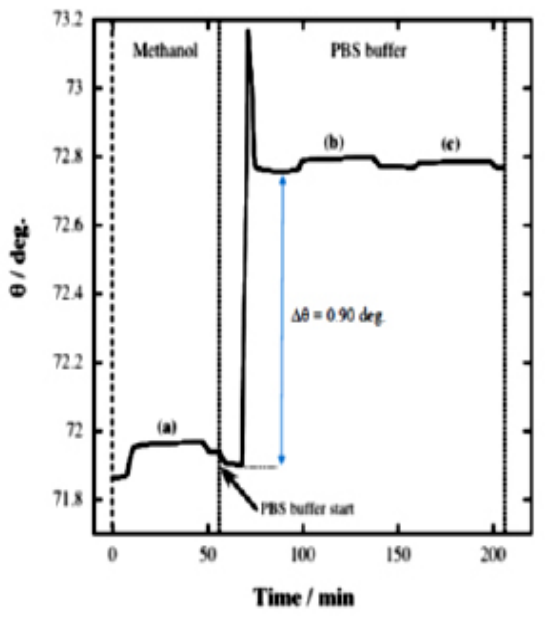

(a)

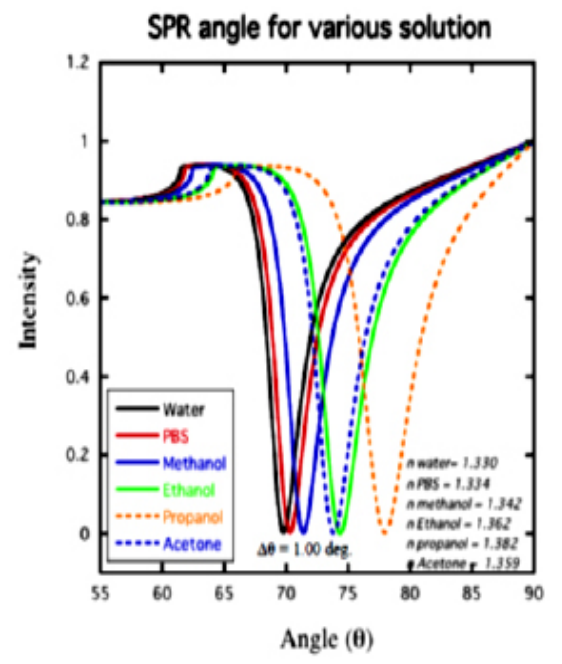

(b)

Fig. 6. SPR sensorgram profile describing the solution effect: (a) SPR sensorgram for surface construction; and (b) SPR angle profile for different solution types using Lab-View SPR program

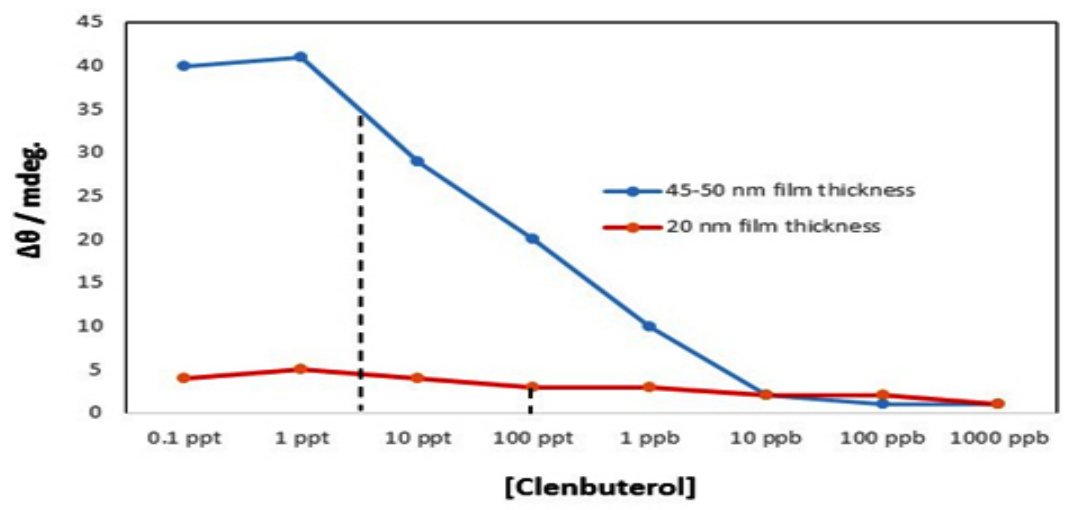

Fig. 7. SPR sensor detection of clenbuterol by indirect competitive inhibition method

\section{CONCLUSION}

The study on metal film thickness and various solvents effect on the SPR biosensor sensitivity for illegal compound detection resulted in some important points. First, the thickness of metal film at about $45-50 \mathrm{~nm}$ showed the best SPR angle shift by better shape and intensity. Second, the solution SPR type in the construction of sensor surface affected on the SPR angle profile, thus, determine the sensitivity in the detection. Finally, the SPR mass transducer sensor surface could be optimized by selecting the best metal thickness at 45-50 nm, thus produce better sensitivity at $5 \mathrm{ppt}$ in the detection of clenbuterol.

\section{ACKNOWLEDGEMENT}

Authors are thankful to the Hitachi Global Foundation for financial support and facilitation during the research activities.

\section{REFERENCES}

1. Dillon, A. C. Chem. Rev., 2010, 110, 6856-6872.

2. Ganeva, J. M. A.; Yao, C.; Feng, Y-L. Talanta., 2014, 130, 475-494.
3. Xu, Y. Environ. Sci. Technol., 2011, 45, 380-385.

4. Hao, C.; Xua, L.; Xing, C.; Kuang, H.;Wang, L.; Xu, C. Biosens. Bioelectron., 2012, 36, 174-178. 
5. Tang, R.; Clark, J. M.; Bond, T.; Graham, N.; Hughes, D.; Freeman, C. Environ. Pollut., 2013, 173, 270-277.

6. Reckmann, K.; Traulsen, I.; Krieter, J. J. Environ. Manag., 2012, 107, 102-109.

7. Blowers, P.; Moline, D. M.; Tetrault, K. F.; Wheeler, R. R.; Tuchawena, S. L. Environ. Sci. Technol., 2008, 42, 1301-1307.

8. Zhang, B.; Fieldman, A.; Wang, Q. Sens. Actuators B., 2013, 186, 597-602.

9. Raj, A.; Lawrence, R.; Lawrence. K.; Silas, N.; Jaless, M.; Srivastava, R. Orient. J. Chem., 2017, 34, 326-335.

10. Johnson, H. O.; Gupta, S.C.;Vecchia, A.V.;Zvomuya, F. J. Environ. Qual., 2009, 38, 1018-1030.

11. O'Connor, G. A.; Elliott, H. A.; Bastian, R. K. J. Environ. Qual., 2008, 37, 157-168.

12. Williams, M. J. Historical Geography., 2000, 26, 28-46.

13. Roehr, A.; Luddecke, K.; Drusch, S.; Mueller, M. J.; Alvensleben, R. V. Food Control ., 2005, 16, 649-655.

14. Rogers, K. R. Anal. Chim. Acta., 2006, 568, 222-231.

15. Rodriguez-Mozaz, S.; Marco, M-P.; Alda, M. J. L. Pure Appl. Chem., 2004, 76, 723-752.

16. Rodriguez-Mozaz, S.; Marco, M-P.; Alda, M.J. L. Barceló, D. Talanta., 2005, 65, 291-297.

17. Thevenot, D. R.; Toth, K.; Durst, R. A.;Wilson, G. S. Biosens. Biolelectron., 2001, 16, 121-131.

18. Rogers,K. R.; Gerlach, C. L. Environ. Sci. Technol., 1996, 30, 486-491.

19. Shankaran, D. R.; Gobi, K. V.; Miura, M. Sens. Actuators B., 2007, 121, 158-177.

20. Bo, B.; Zhu, X.; Miao, P.; Pei, D.; Jiang, B.; Lou, Y.; Shu, Y.; Li, G. Talanta., 2013, 113, 36-40.

21. Riedel, K.; Kunze, G.; Konig, A. Adv. Biochem. Eng. Biotechnol., 2002, 75, 81-118.

22. D'Souza, S. F. Biosens. Bioelectron., 2001, 16, 337-353.

23. Davis, J.; Vaughan, D. H.; Cardosi, M. F. Enzyme Microb. Technol., 1995, 17, 1030-1035.

24. Mulchandani, A.; Bassi, A. S. Crit. Rev. Biotechnol., 1995, 15, 105-124.

25. Crescenzi, C.; Bayoudh, S.; Cormack, P. A. G.; Klein, T.; Ensing, K. Anal. Chem., 2001, 73, 2171-2177.

26. Zhou, H.; Zhang, Z.; He, D.; Xiong, Y. Sens. Actuators B., 2005, 107, 798-804.

27. Liu, S.; Lin, Q.; Zhang, X.; He, X.; Xing, X.; Lian, W.; Huang, J. Sens. Actuators B., 2011, 156, 71-78.
28. Wang, H.; Zhang, Y.; Li, H.; Du, B.; Ma, H.;Wu, D.; Wei, Q. Biosens. Bioelectron., 2013, 49, 14-19.

29. Ho, J-K.; Huo, T-I.; Lin, L-C.; Tsai, T-H. J. Agric. Food Chem., 2014, 62, 9273-9278.

30. Yang, X.; Wu, F.; Chen, D-Z.; Lin, H-W. Sens. Actuators B., 2014, 192, 529-535.

31. Brambilla, G.; Cenci, T.; Franconi, F.; Galarini, R.; Macrì, A.; Rondoni, F.; Strozzie, M.; Loizzo, A. Toxicol. Lett., 2000, 114, 47-53.

32. Mazzanti, G.; Daniele, C.; Boatto, G.; Manca, G.; Brambilla, G.; Loizzo, A. Toxicology., 2003, 187, 91-99.

33. Duan, J.; He, D.; Wang, W.; Liu, Y.; Wu, H.; Wang, Y.; Fu, M.; Li, S. Talanta., 2013, 115, 992-998.

34. Liu, M.; Ning, B.; Qu, L.; Peng, Y.; Dong, J.; Gao, N.; Liu, L.; Gao, Z. Sens. Actuators B., 2012, 161, 124-130.

35. Xiu-Juan, W.; Feng, Z.; Fei, D.; Wei-Qing, L.; Qing-Yu, C.; Xiao-Gang, C.; Cheng-Bao, X.; J. Chromatogr. A., 2013, 1278, 82-88.

36. Sairi, M.; Arrigan, D. W. M. Talanta., 2015 , 132, 205-214.

37. Long, F.; Zhu, A.; Shi, H. Sensors., 2013, 13, 13928-13948.

38. Liang, Y-H.; Chang, C-C.; Chen, C-C.; ChuSu, Y.; Lin, C-W. Clin. Biochem., 2012, 45, 1689-1693.

39. Englebienne, P.; Hoonacker, A. V.; Verhas, M. Spectroscopy., 2003, 17, 255-273.

40. Rich, R. L.; Myszka, D. G. J. Mol. Recog., 2005, 18, 431-478.

41. Karlsson, R. J. Mol. Recog., 2004, 17, 151-161. 42. Winzor, D. J. Anal. Biochem., 2003, 318, 1-12.

43. Homola, J.; Yee, S.; Myszka, D.; in: Ligler, F. S.; Taitt, C. A. R. (Eds.), Surface Plasmon Resonance Biosensors, Elsevier, the Netherlands., 2002, 207-251.

44. Mullett, W. M.; Lai, E. P. C.; Yeung, J. M. Methods., 2000, 22, 77-91.

45. Suherman, S.; Morita, K.; Kawaguchi, T. J. Nonlinear. Opt. Phys., 2016, 25, 165007116500077.

46. Green, R.J.; Frazier, R. A.; Shakesheff, K. M.; Davies, M. C.; Roberts, C. J.; Tendler, S. J. B. Biomaterial., 2000, 21,1823- 1835.

47. Homola, J.; Yee, S. S.; Gauglitz, G. Sens. Actuators B., 1999, 54, 3-15.

48. Suherman, S.; Morita, K.; Kawaguchi, T. Appl. Surf. Sci., 2015, 332, 229-236. 